\title{
Right Ventricular Index for Risk Stratification of Patients with Pulmonary Arterial Hypertension
}

\author{
Christoph Sinning $^{a, c}$ Lars Harbaum ${ }^{b}$ Benedikt Schrage ${ }^{a}$ Nicole Rübsamen $^{a}$ \\ Christina Magnussen $^{a, c}$ Benjamin Waschki ${ }^{a, d}$ Wilko H. Kleemann ${ }^{a}$ Kaaja M. Baaske ${ }^{b}$ \\ Maria Kögler ${ }^{e, f}$ Francisco Ojeda ${ }^{a}$ Christine Fischer ${ }^{g}$ Nicola Benjamin ${ }^{e, f}$ Dirk Westermann ${ }^{a, c}$ \\ Elvin Zengin $^{a} \quad$ Ulrich Schäfer $^{\mathrm{a}} \quad$ Benjamin Egenlaufe, $^{\mathrm{f}}$ Hans F. Klose ${ }^{\mathrm{b}}$ Stefan Blankenberg ${ }^{\mathrm{a}, \mathrm{c}}$ \\ Ekkehard Grünig ${ }^{\mathrm{e}} \mathrm{f}$ \\ ${ }^{a}$ Department of General and Interventional Cardiology, University Heart Center Hamburg, Hamburg, Germany; \\ ${ }^{\mathrm{b}}$ Haematology, BMT with Section Pneumology, University Medical Centre Hamburg-Eppendorf, Hamburg, \\ Germany; ' German Center for Cardiovascular Research (DZHK), Hamburg/Kiel/Lübeck, Germany; ${ }^{\mathrm{d}}$ German Center of \\ Lung Research (DZL), Grosshansdorf, Germany; ${ }^{\mathrm{e} C e n t r e ~ f o r ~ P u l m o n a r y ~ H y p e r t e n s i o n, ~ T h o r a x ~ C l i n i c ~ a t ~ t h e ~ U n i v e r s i t y ~}$ \\ of Heidelberg, Heidelberg, Germany; ${ }^{f}$ German Center of Lung Research (DZL), Heidelberg, Germany; ${ }^{9}$ Department \\ of Human Genetics, University of Heidelberg, Heidelberg, Germany
}

\section{Keywords}

Pulmonary arterial hypertension · Prognosis · Risk stratification · Index · Right heart catheterization · Haemodynamic parameters

\footnotetext{
Abstract

Background: Right ventricular (RV) dysfunction is a major prognostic predictor in pulmonary arterial hypertension (PAH). Objectives: The objective of this study was to assess the prognostic impact of a newly developed index merging haemodynamic parameters into 1 variable. Methods: We retrospectively assessed 2 cohorts of 248 patients (164 from Hamburg and 84 from Heidelberg) with invasively diagnosed PAH. During a median follow-up time of 3.6 years (3.1 and 4.0 years for Hamburg and Heidelberg, respectively), the composite endpoint of all-cause mortality and lung transplantation occurred in 57 patients (53 and 4 patients for Hamburg and Heidelberg, respectively). The RV index was
}

developed in the Hamburg cohort and validated in the Heidelberg cohort: (right atrial pressure $\times$ pulmonary vascular resistance)/mixed venous oxygen saturation. Results: Patients with a high RV index had a higher incidence of the combined endpoint in Kaplan-Meier analyses in the Hamburg and Heidelberg cohort $(p=0.017$ and $p=0.034$, respectively). The calculated RV index cut-off value was 91 and identified patients with a worse outcome in the Hamburg cohort and showed a trend in the Heidelberg cohort $(p<$ 0.001 and $p=0.089$, respectively). The RV index in Cox regression hazard models was an independent predictor of outcomes after adjustment for sex and age in both cohorts (Hamburg: hazard ratio [HR] 1.26 [95\% Cl 1.08, 1.47], $p=$ 0.0027; Heidelberg: HR 2.27 [95\% Cl 1.46, 3.51], $p<0.001$ ). A

C.S., L.H. and B.S. contributed equally as first authors.

H.F.K. and S.B. are equal senior authors.

All authors take responsibility for all aspects of the reliability and freedom from bias of the data presented and their discussed interpretation.

\section{KARGER}

(c) 2018 S. Karger AG, Basel

E-Mail karger@karger.com

www.karger.com/res
Christoph Sinning, MD

Department of General and Interventional Cardiology

University Hospital Hamburg-Eppendorf

Martinistrasse 52, DE-20246 Hamburg (Germany)

E-Mail c.sinning@uke.de 
nomogram based on these results allowed risk stratification. Conclusion: Merging 3 haemodynamic variables into 1 variable, the RV index increased the prognostic power up to an independent risk factor. The RV index is easy to calculate and allows the construction of a nomogram for an individualized risk assessment.

(c) 2018 S. Karger AG, Basel

\section{Introduction}

An important improvement in the management of pulmonary arterial hypertension (PAH) was the change in treatment algorithm proposed by the 2015 European Guidelines for Pulmonary Hypertension $(\mathrm{PH})$ with an early intensified combination treatment in severely diseased patients [1]. For this strategy, which is based on a number of randomized, controlled, double-blinded trials, a dynamic risk stratification has been recommended $[2,3]$.

In a recent study analysing data from patients with newly diagnosed PAH enrolled into COMPERA, a European-based $\mathrm{PH}$ registry, the risk estimates proposed in the European $\mathrm{PH}$ guidelines with annual mortality risks of $<5,5-10$ and $>10 \%$ in patients at low, intermediate or high risk, respectively, were confirmed, both at baseline as well as at follow-up [2]. An abbreviated version of the risk assessment strategy was applied, assessing the noninvasive and invasive variables WHO functional class, 6-min walking distance (6MWD), N-terminal fragment of B-type natriuretic peptide (NT-proBNP), right atrial pressure (RAP), cardiac index and mixed venous oxygen saturation $\left(\mathrm{SvO}_{2}\right)$ in 879 patients. These data confirmed findings of a study which used a similar subset of variables in 530 Swedish patients with newly diagnosed PAH [4]. However, in a new study rom the French PAH registry, only a $6 \mathrm{MWD} \leq 440 \mathrm{~m}$ independently predicted death or transplantation, whereas baseline WHO/NYHA functional class III-IV, RAP $\geq 8 \mathrm{~mm} \mathrm{Hg}$ and cardiac index $<2.5 \mathrm{~L} \times \mathrm{min}^{-1} \times \mathrm{m}^{-2}$ were associated with a higher risk of death or transplantation in the univariate analysis [5]. These and further studies [4-7] confirmed that haemodynamic variables obtained by right heart catheterization are essential for risk stratification as proposed by the guidelines. On the other hand, in many studies, single haemodynamic variables were not independent prognostic predictors and varied largely in their prognostic relevance within different studies $[2,4-6,8]$.

Therefore, we aimed to develop an index based on a composition of invasive variables to improve their prog- nostic power in the context of non-invasive predictors. The right ventricular (RV) index is a combined index consisting of the variables with the highest impact regarding prognostic outcome; thus, RAP and pulmonary vascular resistance (PVR) are in the enumerator and $\mathrm{SvO}_{2}$ in the denominator position. Furthermore, we aimed to compare the newly developed RV index with previous prognostic equations to identify patients with a poor outcome and the need for intensification of treatment.

\section{Methods}

Study Population and Design

We retrospectively reviewed all consecutive patients presenting for right heart catheter (RHC) and aged $\geq 18$ years with idiopathic, heritable or associated PAH who were seen in the centres of Hamburg (between February 2010 and June 2016) and Heidelberg (between January 2014 and June 2016). Patients with prevalent disease and newly diagnosed patients were enrolled. Inclusion criteria required a baseline RHC confirming $\mathrm{PAH}$, defined as a mean pulmonary arterial pressure $(\mathrm{mPAP}) \geq 25 \mathrm{~mm} \mathrm{Hg}$, a pulmonary arterial wedge pressure $\leq 15 \mathrm{~mm} \mathrm{Hg}$ and a pulmonary vascular resistance $>3$ Wood units [1]. Patients were excluded if they lacked a calculable follow-up time or a complete re-evaluation with physical examination, a 6MWD, NT-proBNP measurement and RHC, including all haemodynamic variables at baseline (time of diagnosis). All data were anonymized and compiled according to the requirements of the local ethics committees and the Declaration of Helsinki.

The combined primary endpoint of the study was all-cause death and lung transplantation and was assessed using the electronic patient record systems of the participating hospitals.

\section{RHC Measurement}

RHC was performed as previously described [9]. In brief, the jugular vein was punctured with ultrasound guidance, and a SwanGanz catheter was inserted for pressure measurements and blood samples. The pressure was equalized at the mid-thoracic line in the supine patient. Pressure measurements were done at the end of expiration.

Important Non-Invasive Variables for Risk Stratification

The 6MWD was performed under standardized conditions [10], and serum NT-proBNP was obtained at baseline.

\section{Statistical Methods}

Comparison of the 2 Cohorts

Continuous variables were described as means \pm standard deviations and categorical variables as absolute numbers and percentages. The $t$ test (for continuous variables) or the $\chi^{2}$ test (for categorical variables) were employed for between-group comparisons.

Additional analysis consisted of 4 steps: (1) development of an index (called "RV index") to predict the combined endpoint in the Hamburg cohort and assessment of its predictive ability; (2) finding an optimal cut point of the index in the Hamburg cohort; (3) validation of the index and the cut point in the Heidelberg cohort; and (4) comparison of predictive ability between RV index and the 
already published (a) FPHN ItinérAIR-HTAP predictive equation [3] and (b) NIH risk stratification equation adapted by Thenappan et al. [11] in the validation cohort.

Selection of the Variables for the RV Index and Its Additional Information besides Single Haemodynamic Variables

Associations between the single haemodynamic parameters and the combined endpoint were assessed by unadjusted Cox regression models. Fractional polynomials ("mfp" package of R [12]) were used to explore the presence of non-linear relationships between the haemodynamic parameters and the combined endpoint. The index was built by multiplying all haemodynamic parameters that showed significant $(p<0.05)$ associations in the univariate screening. Depending on the direction of the association, the parameters were entered as reciprocals (if hazard ratio $[\mathrm{HR}]<1$ ) or without transformation (if $\mathrm{HR}>1$ ) into the index. All further analyses included only patients with a non-missing index. The predictive ability of the index was assessed by calculating 3 multivariate Cox regression models that included the index and non-haemodynamic adjustment variables: model 1 adjusted for age and sex; model 2 adjusted for age, sex and $\log (\mathrm{NT}$-proBNP); and model 3 adjusted for age, sex, $\log (\mathrm{NT}$-proBNP) and 6MWD. These adjustment variables were used because they are known to be associated with mortality. Model 2 and 3 were used to investigate the predictive ability of the index in the presence of the predictors already in use (NT-proBNP and 6MWD). Interactions between RV index and each of the variables age, sex, NT-proBNP or 6MWD were added to model 3 and tested for significance $(p<0.1)$.

Concordance indices ( $\mathrm{C}$ index) for the RV index and each of its components (adjusted for all non-haemodynamic adjustment variables that showed significant associations with the combined endpoint) were compared to investigate if all components were necessary for the index.

Outcome Analysis

Analysis of the combined outcome was compared between groups either using the log-rank test or, in case of violation of the proportional hazards assumption, using the Gehan-Breslow test. An optimal cut point for the index was found by applying the method of Williams et al. [13]. The predictive ability of the dichotomized index was assessed using adjusted Cox regression models and Kaplan-Meier curves (as described above).

\section{Validation of the RV Index in the Heidelberg Cohort}

The predictive ability of the RV index, used both as a continuous and a dichotomized variable, was assessed in the Heidelberg cohort by calculating the 3 Cox regression models and KaplanMeier curves as described above. Calibration of models including either the continuous or the dichotomized RV index was assessed in both cohorts separately ("rms" package of R [14]).

A nomogram was created to provide a ready-to-use version of the continuous index based on data combined from both cohorts. The nomogram included the RV index and all non-haemodynamic adjustment variables that showed significant associations with the combined endpoint.

Comparison with the FPHN ItinérAIR-HTAP Predictive Equation and the NIH Risk Stratification Equation

To compare the nomogram with the FPHN ItinérAIR-HTAP predictive equation [3] and the NIH risk stratification equation
[11] regarding their ability to predict 2 -year event-free survival, C indices (separately for each equation) were calculated in the validation cohort. In the FPHN ItinérAIR-HTAP predictive equation, the survival at year $\mathrm{t}$ is computed as $\exp (-0.02-0.28 \times \mathrm{t})^{\mathrm{A}(\mathrm{x}, \mathrm{y}, \mathrm{z})}$ with $\mathrm{A}(\mathrm{x}, \mathrm{y}, \mathrm{z})=\exp (-(0.004 \times(6 \mathrm{MWD}-280)+0.98 \times$ (indicator if female $)+0.28 \times($ cardiac output -4$)$. In the NIH risk stratification equation, the survival at year $\mathrm{t}$ is computed as $\exp (-\mathrm{A}(\mathrm{x}, \mathrm{y}, \mathrm{z})$ $\times \mathrm{t})$ with $\mathrm{A}(\mathrm{x}, \mathrm{y}, \mathrm{z})=\exp (-1.270-0.0148 \times \operatorname{mPAP}+0.0402 \times$ RAP $-0.361 \times$ cardiac index $)$. All computations were performed with $\mathrm{R}$ (version 3.4.1).

\section{Results}

\section{Study Population with Baseline Characteristics}

We included 164 patients in the Hamburg and 84 patients in the Heidelberg cohort fulfilling the inclusion criteria. The Hamburg cohort consisted of prevalent and newly diagnosed cases $(n=68 / 96)$, and the Heidelberg cohort included treatment-naive, newly diagnosed patients only. Patients with idiopathic and hereditary PAH formed the largest subgroup in both cohorts $(71 \%$ in Hamburg and $67 \%$ in Heidelberg), followed by patients with drug-induced PAH (1\% in Hamburg and 0 in Heidelberg) and patients with associated forms of PAH (19\% in Hamburg and 22\% in Heidelberg). The characteristics of these patients are shown in Table 1. In general, neither classification into subtypes nor associated diseases could be shown to be differently distributed between cohorts $(p=0.7$ and $p=0.12$, respectively). Patients in the Heidelberg cohort received more medication, except for prostacyclin agonists, which were evenly distributed $(p=0.43)$. No differences were seen for median age (Hamburg: 63.9 years, range 51.7-73.6; Heidelberg: 70.0 years, range $55.0-74.0)$ or sex (68.3\% men in the Hamburg and $61.9 \%$ men in the Heidelberg cohort). Both cohorts differed in terms of cardiac output and $\mathrm{SvO}_{2}$, which were higher in the Heidelberg sample $(p<0.001$ and $p=0.043$, respectively), whereas PVR was higher in the Hamburg cohort $(p=0.034)$. RV index did not differ between cohorts (Table 1).

\section{Mortality in the Development and Validation Cohort}

During a median follow-up time of 3.6 years (3.1 and 4.0 years for Hamburg and Heidelberg, respectively) the combined endpoint of all-cause mortality or lung transplantation occurred in 57 patients (53 and 4 patients for Hamburg and Heidelberg, respectively); 4 patients underwent lung transplantation. The survival curves in the cohorts from Hamburg and Heidelberg were similar (Fig. 1) and showed 1-, 2- and 5-year event rates of 12.2, 18.2 and 
28.8\% in Hamburg and 2.5, 11.0 and 29.2\% in Heidelberg. The 1-, 2- and 5-year survival rates were $87.8,81.8$ and 71.2\% in Hamburg and 97.5, 89.0 and 70.8\% in Heidelberg.

\section{Development of the RV Index and Application in the} Development Cohort

In the univariate analysis of the haemodynamic parameters obtained in the Hamburg cohort, RAP, PVR and
$\mathrm{SvO}_{2}$ were significant predictors of prognosis, whereas PAPs (systolic PAP, diastolic PAP and mPAP) and cardiac output (cardiac output and cardiac index) were not. Thus, the RV index was constructed using these 3 parameters as $\mathrm{RAP} \times \mathrm{PVR} / \mathrm{SvO}_{2}$. The combined outcome was more common with rising RV index as shown in Figure 2. Patients with an RV index in the highest third had a worse prognosis in both cohorts compared to those with an RV

Table 1. Demographics and baseline characteristics of the study population

\begin{tabular}{|c|c|c|c|}
\hline & $\begin{array}{l}\text { Hamburg } \\
(n=164)\end{array}$ & $\begin{array}{l}\text { Heidelberg } \\
(n=84)\end{array}$ & $p$ value \\
\hline $\mathrm{RV}$ index & $164.8 \pm 432.6$ & $97.8 \pm 98.1$ & 0.16 \\
\hline Age at right heart catheter, years & $61.7 \pm 14.7$ & $63.6 \pm 14.0$ & 0.33 \\
\hline Male sex & $112(68.3)$ & $52(61.9)$ & 0.39 \\
\hline BMI & $27.5 \pm 6.1$ & $28.2 \pm 5.5$ & 0.38 \\
\hline Incident $\mathrm{PAH}$ & $96(59)$ & $84(100)$ & $<0.001$ \\
\hline Prevalent PAH & $68(41)$ & - & \\
\hline PAH diagnosis & & & 0.70 \\
\hline Idiopathic & $110(67)$ & $52(62)$ & \\
\hline Heritable & $5(3)$ & $3(4)$ & \\
\hline Drug-induced & $2(1)$ & $0(0)$ & \\
\hline PAH associated with & $47(29)$ & $29(34)$ & 0.12 \\
\hline Connective tissue & $31(19)$ & $19(22)$ & \\
\hline HIV related & $3(2)$ & $0(0)$ & \\
\hline Portopulmonary & $6(4)$ & $1(1)$ & \\
\hline Congenital heart disease & $7(4)$ & $9(11)$ & \\
\hline Acute vasodilator responder & $5(3)$ & $7(8)$ & 0.07 \\
\hline WHO/NYHA functional class & & & 0.29 \\
\hline I/II & $23(14)$ & $20(24)$ & \\
\hline III & $116(70)$ & $57(68)$ & \\
\hline IV & $25(16)$ & $7(8)$ & \\
\hline \multicolumn{4}{|l|}{ Medication } \\
\hline Number of PAH drugs & $1.2 \pm 0.9$ & $1.7 \pm 0.7$ & $<0.001$ \\
\hline PDE5 inhibitor & $86(52.4)$ & $66(78.6)$ & $<0.001$ \\
\hline Endothelin receptor antagonist & $84(51.2)$ & $57(67.9)$ & 0.018 \\
\hline Prostacyclin agonists & $24(14.8)$ & $9(10.7)$ & 0.43 \\
\hline Soluble guanylate cyclase stimulator & $4(2.4)$ & $10(11.9)$ & 0.0061 \\
\hline \multicolumn{4}{|l|}{ Hemodynamic variables } \\
\hline mPAP, mm Hg & $45.0 \pm 15.7$ & $44.3 \pm 14.1$ & 0.73 \\
\hline $\mathrm{RAP}, \mathrm{mm} \mathrm{Hg}$ & $8.9 \pm 5.7$ & $7.7 \pm 4.5$ & 0.079 \\
\hline $\mathrm{CO}, \mathrm{L} / \mathrm{min}$ & $3.9 \pm 1.6$ & $4.6 \pm 1.3$ & $<0.001$ \\
\hline $\mathrm{SvO}_{2}, \%$ & $60.7 \pm 12.3$ & $63.9 \pm 9.8$ & 0.043 \\
\hline PVR, Wood units & $9.93 \pm 6.82$ & $8.2 \pm 4.24$ & 0.034 \\
\hline Heart rate, bpm & $76.9 \pm 13.7$ & $79.0 \pm 13.3$ & 0.24 \\
\hline Systolic RR, mm Hg & $128.4 \pm 21.9$ & $120.4 \pm 16.1$ & 0.0038 \\
\hline Diastolic RR, mm Hg & $74.9 \pm 14.8$ & $73.8 \pm 9.8$ & 0.53 \\
\hline
\end{tabular}

Values are means \pm standard deviations or $n(\%)$. BMI, body mass index; PAH, pulmonary arterial hypertension; mPAP, mean pulmonary arterial pressure; RAP, right atrial pressure; $\mathrm{CO}$, cardiac output; $\mathrm{SvO}_{2}$, mixed venous oxygen saturation; PVR, pulmonary vascular resistance; RR, blood pressure. 
index in the lowest tertile (Hamburg: HR 1.47 [95\% CI 1.04, 2.08]; Heidelberg: HR 1.85 [95\% CI 0.89, 3.86]).

The optimal cut point for the RV index for the timeto-event outcome in the Hamburg cohort was 91. The survival curves for the combined endpoint by the dichotomized RV index are shown in Figure 3. In both cohorts, patients with an RV index $>91$ had a significantly increased mortality compared to patients with an RV index $\leq 91(p=0.00092)$. The fully adjusted Cox regression analysis (model 3 ) showed a more than 2.5 -fold higher mortality risk in patients with an RV index above the cutoff (HR 2.56 [95\% CI 1.29, 5.08], $p=0.0071$; Fig. 4).

\section{Cox Regressions for Continuous and Dichotomized $R V$ Index in the Hamburg Cohort}

Age- and sex-adjusted multivariate Cox regressions showed significant associations for the RV index and the combined endpoint $(p=0.0027)$ which persisted after additional adjustment for NT-proBNP or NT-proBNP and 6MWD (Fig. 4). No significant interactions between RV index and age, sex, NT-proBNP or 6MWD were found. Besides RV index, age was the only significant independent predictor of the combined endpoint in the multivariate analyses (online suppl. Table 1; for all online suppl. material, see www.karger.com/doi/10.1159/000489231).

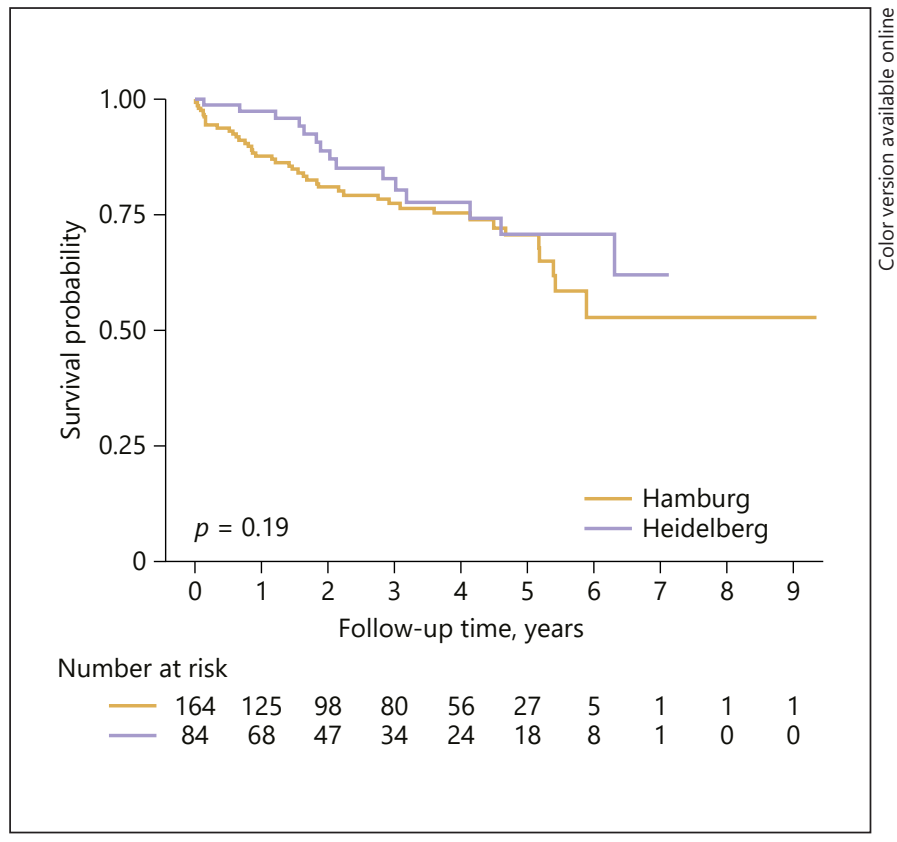

Fig. 1. Kaplan-Meier curve analysis for the derivation (Hamburg) and validation (Heidelberg) cohort for the combined endpoint allcause death and lung transplantation according to tertiles of the $\mathrm{RV}$ index. RV, right ventricular.

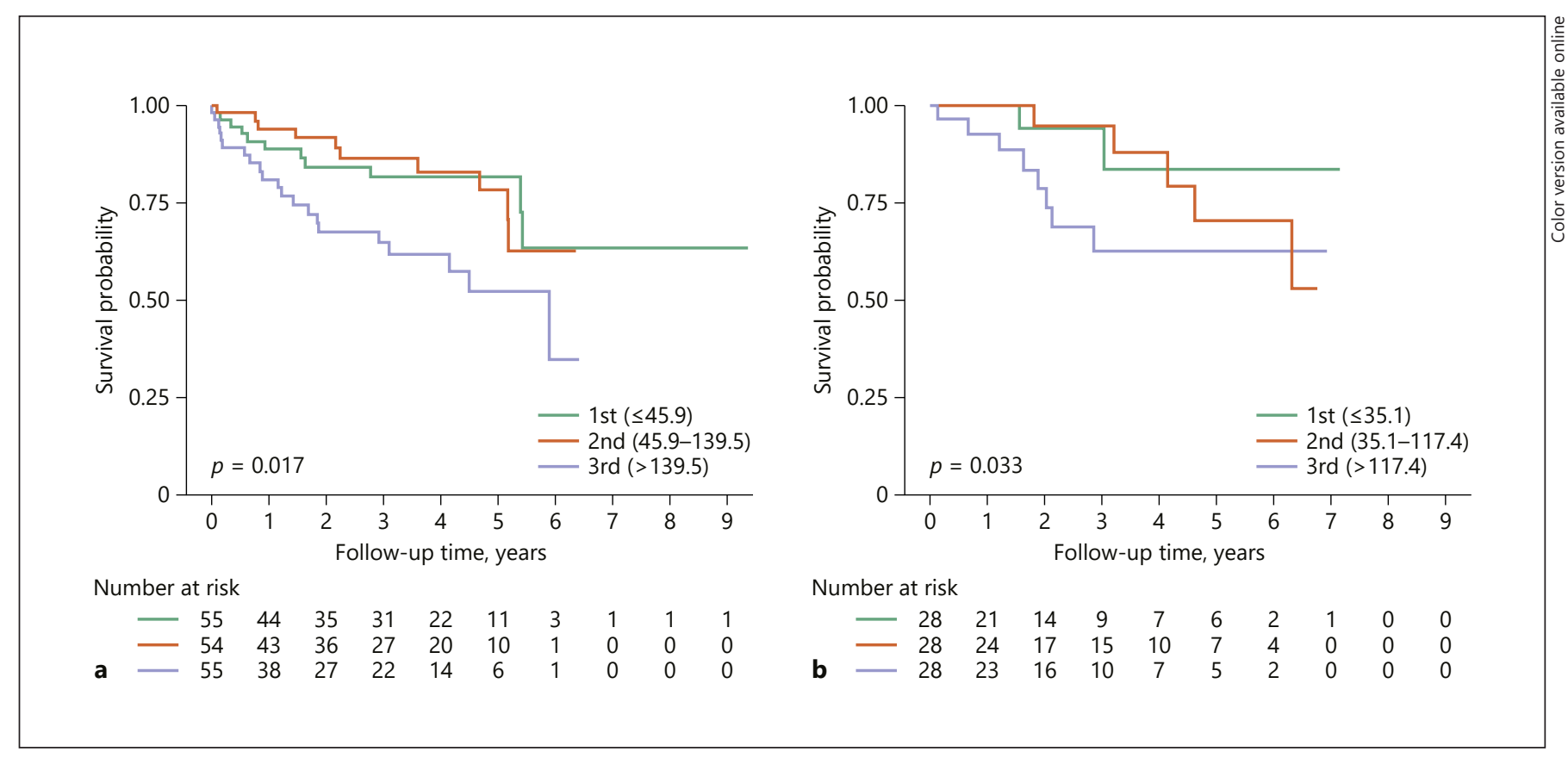

Fig. 2. Kaplan-Meier curve analysis for the combined endpoint all-cause death and lung transplantation by tertiles of the RV index comparing the derivation (a, Hamburg) and validation (b, Heidelberg) cohort. RV, right ventricular. 

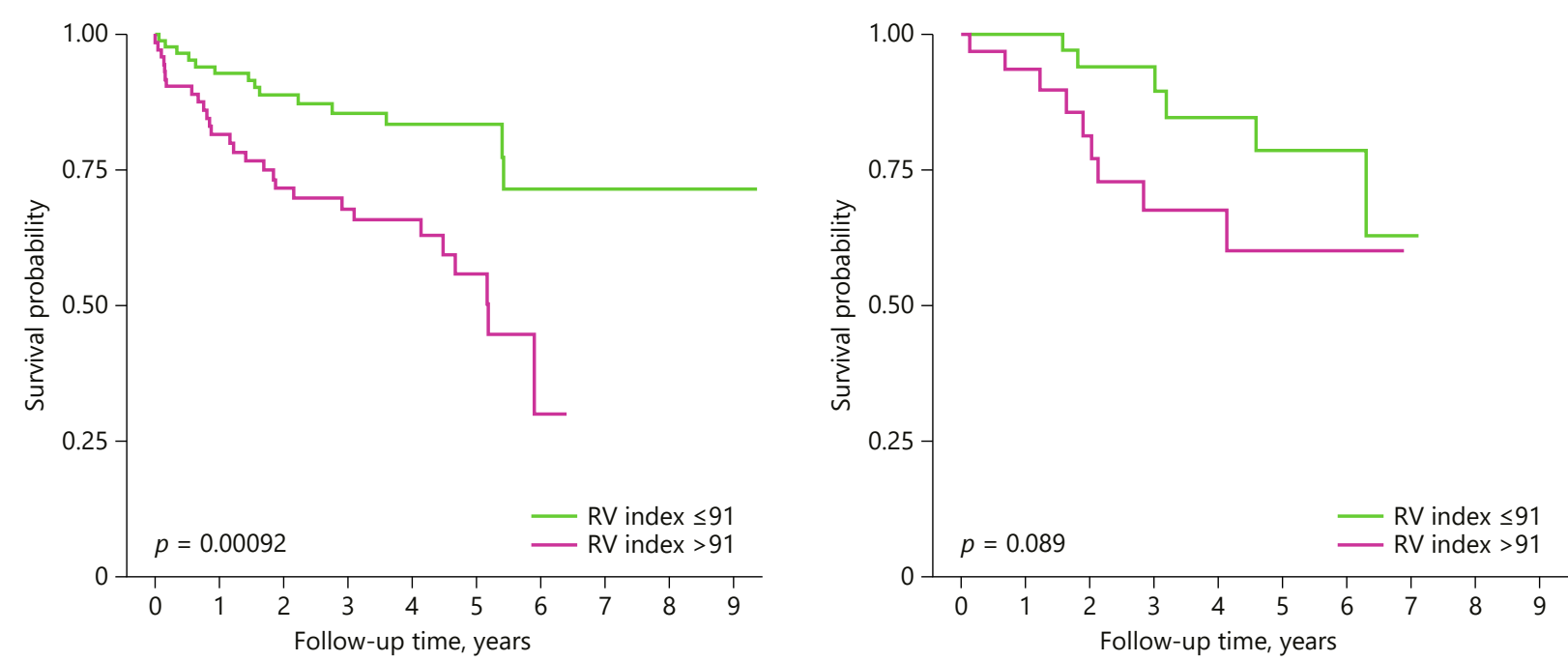

Number at risk

$\begin{array}{lllllllllll}\mathbf{a} & -90 & 73 & 58 & 48 & 33 & 17 & 4 & 1 & 1 & 1 \\ & 74 & 52 & 40 & 32 & 23 & 10 & 1 & 0 & 0 & 0\end{array}$

Number at risk

b $\quad \begin{array}{rlllllllll}52 & 41 & 28 & 21 & 15 & 12 & 5 & 1 & 0 & 0 \\ 32 & 27 & 19 & 13 & 9 & 6 & 3 & 0 & 0 & 0\end{array}$

Fig. 3. Kaplan-Meier curve analysis of the application of the calculated cut point of the RV index for the Hamburg (a) and Heidelberg (b) cohorts. RV, right ventricular.

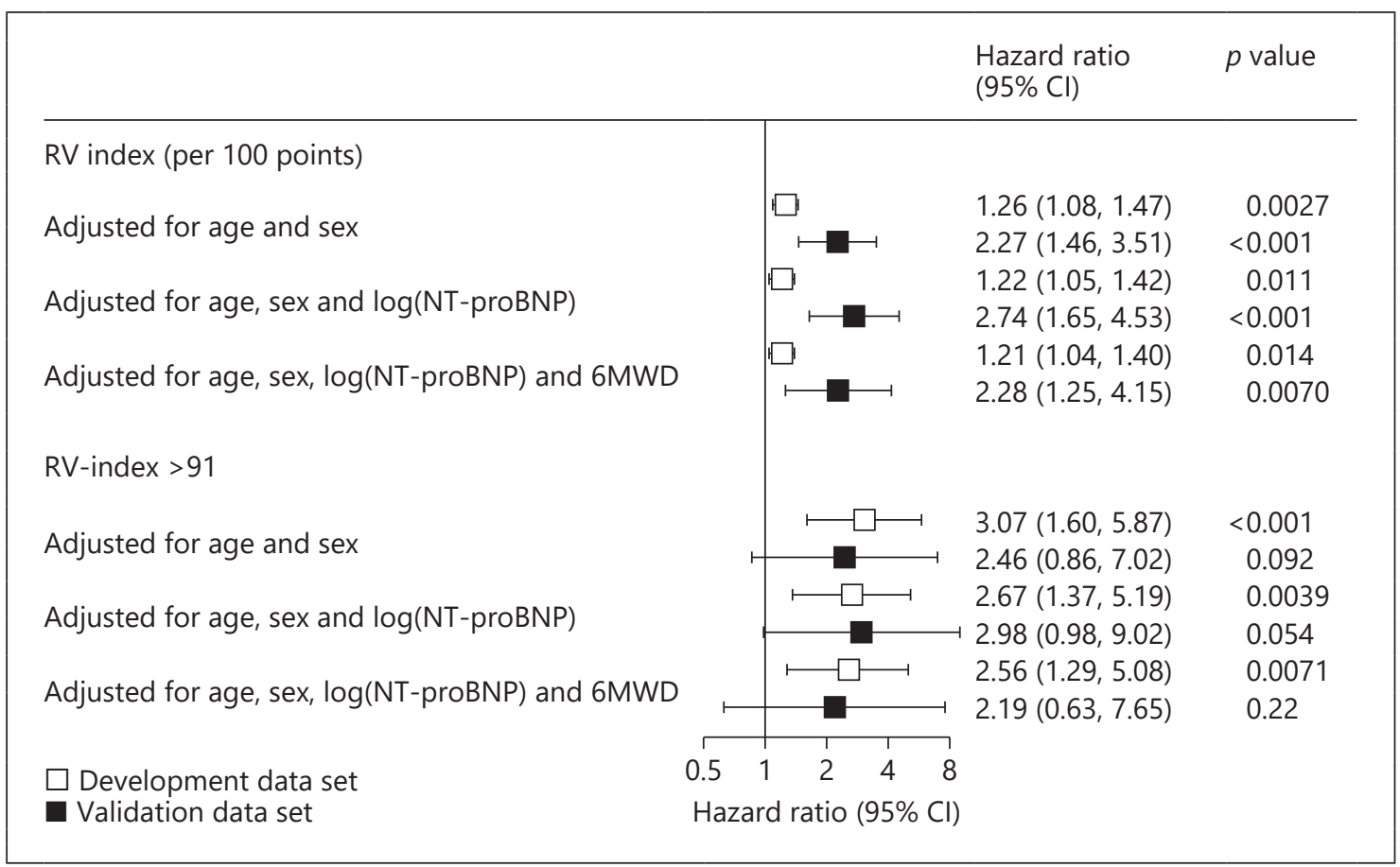

Fig. 4. Forest plot of all Cox regression analyses performed with the RV index. Model 1 was adjusted for age and sex, model 2 for age, sex and $\log (\mathrm{NT}$-proBNP) and model 3 for age, sex, $\log (\mathrm{NT}$-proBNP) and 6MWD. RV, right ventricular; NT-proBNP, N-terminal fragment of B-type natriuretic peptide; 6MWD, 6-min walking distance. 


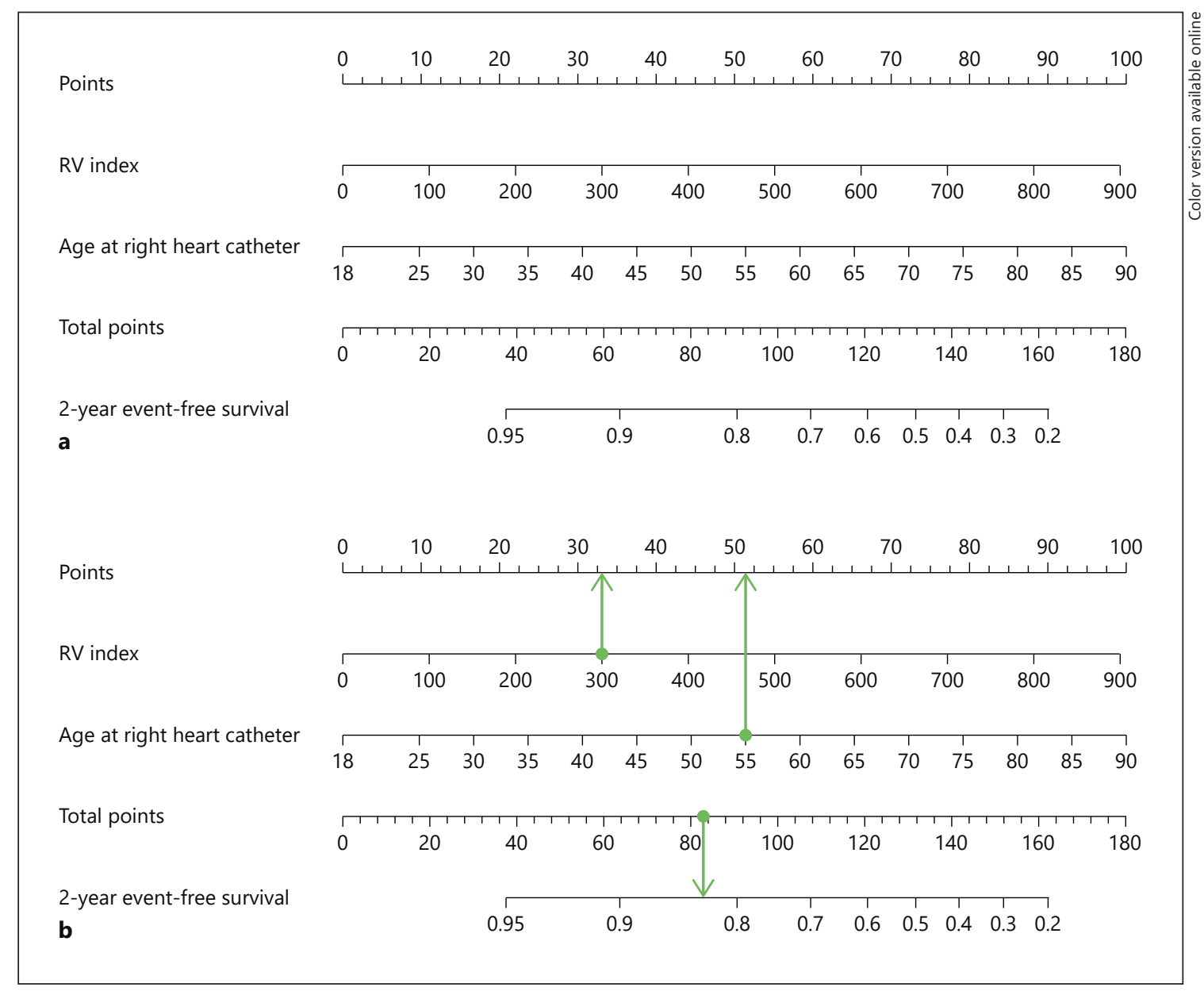

Fig. 5. a Nomogram for establishing the 2-year risk for an event in the study population. b For example, a 55-yearold patient with an RV index of 300 receives 51 points for age and 32 points for the index value, summing up to 83 points. A result of 83 points indicates a probability of $82 \%$ to have a 2 -year event-free survival.

Prognostic Information of Single and Combined

Haemodynamic Variables

The RV index had a higher $C$ index than each of its components $(0.699$ vs. 0.639 for PVR, 0.664 for RAP and 0.640 for $\mathrm{SvO}_{2}$; all adjusted for age and corrected for overoptimism). Age alone had a C index of 0.620 .

\section{Validation in the Heidelberg Cohort}

In the Heidelberg cohort, Cox regressions for RV index and survival showed similar results as in the Hamburg sample (Fig. 3). However, only a trend towards a higher mortality risk was found in Cox regressions for the dichotomized RV index and survival, but these associations missed statistical significance (HR 2.19 [95\% CI 0.63, 7.65] in model 3, $p=0.22$ ). The results for using the cut point in the Heidelberg cohort are shown in Figure $3 b$.

\section{Individual Risk Stratification Using a Nomogram}

A nomogram accounting for both variables predicting the outcome of the multivariate analysis is shown in Figure 5, which can be used for risk stratification in the examined patient cohorts. Furthermore, in Figure 5, an example is given to illustrate how the nomogram is applied to a patient in the real-world setting.

\section{Comparison of Risk Stratification Equations in the}

\section{Validation Cohort}

The $\mathrm{C}$ index of the survival probability according to the RV index nomogram was 0.851 compared to 0.76 for the FPHN ItinérAIR-HTAP predictive equation and 0.712 for the NIH risk stratification equation when the derived RV index was applied in the validation cohort. 


\section{Discussion}

To the best of our knowledge, this study demonstrated for the first time that the prognostic value of the invasively measured haemodynamic parameters RAP, PVR and $\mathrm{SvO}_{2}$ was increased in patients with $\mathrm{PAH}$ when these parameters were merged as an RV index using the simple formula $\mathrm{RAP} \times \mathrm{PVR} / \mathrm{SvO}_{2}$. The study was designed to use 2 large PAH treatment cohorts for the development (Hamburg) and the validation (Heidelberg) of results to show the additional benefit of the RV index for risk prediction. In addition, a high RV index (>91) identified $\mathrm{PAH}$ patients with an increased risk for the combined endpoint mortality and lung transplantation and was an independent risk factor after adjustment for age, sex, 6MWD and NT-proBNP. The RV index could also be used in a nomogram for individual risk stratification and may be a useful tool for prognostic assessment in PAH patients. Results for the RV index were at least as good as the previously suggested FPHN ItinérAIR-HTAP predictive equation [3] and the NIH risk stratification equation [11] which were, however, more complicated to calculate and are, therefore, not in widespread use in clinical practice.

\section{Haemodynamic Variables and Risk Stratification in $P A H$}

Although risk stratification is possible with non-invasive measures especially in the low-risk group $[2,4,6,8]$, haemodynamic values, as measured with RHC, are recommended in the current guidelines for risk stratification $[6,7]$. However, there is still a debate about which parameters are the best to be used for this approach as studies report different results regarding single haemodynamic parameters $[2,4-6,8]$. The current guidelines recommend RAP, cardiac index and $\mathrm{SvO}_{2}$ as variables for risk prediction [1]. However, while RAP was most commonly reported to be associated with the outcome $[2,4-6]$, this was not true for the cardiac index in 2 large recent studies $[5,8]$. One of these studies [5] suggested that stroke volume index as a predictor was superior to other haemodynamic variables reflecting cardiac function; however, in the same study, $\mathrm{SvO}_{2}$ as a reportedly strong predictor of outcome $[4,15]$ was lacking because of missing data. The second study [8] did not test the additional use of $\mathrm{SvO}_{2}$, although the data were present. The additional use of PVR in our cohort was not commonly under investigation in the described studies but was evaluated in the large REVEAL registry [16] and was of additional use to detect patients with the outcome. Thus, our reported findings were in line with the current literature regarding RAP and $\mathrm{SvO}_{2}$, which were commonly used; however, PVR was not so often a focus of research, although data of a large registry speak in favour of this measure. In this context, it has to be stressed that each haemodynamic parameter is useful regarding pathophysiological considerations; however, the combination in an RV index might be better suited to identify patients with an adverse outcome.

\section{Combination of Haemodynamic Variables and Use of a Cut Point}

None of the previous studies, however, did use a combined model of haemodynamic variables to improve risk prediction, which was shown in our cohort with an increased C index for the RV index in comparison to each of the variables alone. Especially the suggested cut point with an RV index of 91 might be useful in the clinical setting, as patients below the cut point had a significantly lower rate of events. The RV index had a higher $\mathrm{C}$ index, which is a global validation of the predictive ability of a survival model [17].

\section{Individual Risk Stratification with the RV Index}

In addition, it has to be pointed out that the RV index allocates every patient to just 1 value, and it allows the use of a nomogram which can calculate an individual risk. Thus, in our study, only 59 patients out of 248 could be allocated to a distinct category by applying the recommended variables and risk category scheme from the 2015 $\mathrm{PAH}$ guidelines because the current scheme uses categorization of continuous variables, limiting the information for risk prediction.

\section{Haemodynamic Variables as Outcome and Selection Variables in Current Studies}

Regarding the combination of the included variables in the RV index, recent results of the SERAPHIN study could show the impact of RAP [5] on outcomes due to the association with increased PVR and worsening right heart function [6]. In addition, cardiac index is decreased in patients with severe PAH as a result of the PVR, as was also shown in our results [6]. Furthermore, in the SERAPHIN as well as in another treatment study, haemodynamic variables were used to select certain patient collectives and as outcome measures to show the effect of treatment $[6,7,18]$.

\section{Limitations}

A limitation of this study was its retrospective design analysing a mixed cohort of patients in Hamburg with 
prevalent and incident $\mathrm{PAH}$, whereas the Heidelberg cohort included newly diagnosed, untreated incident PAH patients only. Nevertheless, the newly developed RV risk score has been evaluated with a second study cohort, which is a strength of the study. However, further prospective studies are needed to confirm the results, including defined follow-up assessments using a second right heart catheterization.

\section{Conclusion}

The results of this study indicated that calculating an RV index out of 3 haemodynamic standard variables (RAP, PVR and $\mathrm{SvO}_{2}$ ) obtained by RHC increased their prognostic power. Using this formula, the RV index was the only independent risk factor (beside age) among clinically relevant parameters. Furthermore, the RV index allowed to construct a nomogram for an individual risk stratification.
The RV index had a higher $\mathrm{C}$ index regarding the reported outcome in our validation cohort than previously reported risk prediction equations, which are, however, cumbersome to use in clinical practice. Yet, the results of this study have to be validated in further prospective studies including defined follow-up assessments using a second RHC.

\section{Disclosure Statement}

Prof. Blankenberg reports grants and personal fees from Abbott, Abbott Diagnostics, Bayer, Boehringer Ingelheim, Thermo Fisher and Siemens and personal fees from Medtronic, Pfizer, Roche, Siemens Diagnostics and Novartis outside the submitted work. Prof. Grünig reports grants and personal fees from Actelion and Bayer/MSD, personal fees from SCOPE and grants from GSK, United Therapeutics, Novartis, Pfizer, REATA and Bellerophon outside the submitted work. Dr Klose reports grants and personal fees from Actelion and GSK, personal fees from MSD, Bayer and United Therapeutics outside the submitted work. The other authors have no potential conflicts of interest to disclose.

\section{References}

1 Galie N, Humbert M, Vachiery JL, Gibbs S, Lang I, Torbicki A, Simonneau G, Peacock A, Vonk Noordegraaf A, Beghetti M, Ghofrani A, Gomez Sanchez MA, Hansmann G, Klepetko W, Lancellotti P, Matucci M, McDonagh T, Pierard LA, Trindade PT, Zompatori M, Hoeper M, Aboyans V, Vaz Carneiro A, Achenbach S, Agewall S, Allanore Y, Asteggiano R, Paolo Badano L, Albert Barbera J, Bouvaist H, Bueno H, Byrne RA, Carerj S, Castro G, Erol C, Falk V, Funck-Brentano C, Gorenflo M, Granton J, Iung B, Kiely DG, Kirchhof P, Kjellstrom B, Landmesser U, Lekakis J, Lionis C, Lip GY, Orfanos SE, Park MH, Piepoli MF, Ponikowski P, Revel MP, Rigau D, Rosenkranz S, Voller H, Luis Zamorano J: 2015 ESC/ ERS Guidelines for the diagnosis and treatment of pulmonary hypertension: the Joint Task Force for the Diagnosis and Treatment of Pulmonary Hypertension of the European Society of Cardiology (ESC) and the European Respiratory Society (ERS): endorsed by: Association for European Paediatric and Congenital Cardiology (AEPC), International Society for Heart and Lung Transplantation (ISHLT). Eur Heart J 2016;37:67-119.

2 Hoeper MM, Kramer T, Pan Z, Eichstaedt CA, Spiesshoefer J, Benjamin N, Olsson KM, Meyer K, Vizza CD, Vonk-Noordegraaf A, Distler O, Opitz C, Gibbs JSR, Delcroix M, Ghofrani HA, Huscher D, Pittrow D, Rosenkranz S, Grunig E: Mortality in pulmonary arterial hypertension: prediction by the 2015 European pulmonary hypertension guidelines risk stratification model. Eur Respir J 2017;50:pii1700740.
3 Sitbon O, Benza RL, Badesch DB, Barst RJ, Elliott CG, Gressin V, Lemarie JC, Miller DP, Muros-Le Rouzic E, Simonneau G, Frost AE, Farber HW, Humbert M, McGoon MD: Validation of two predictive models for survival in pulmonary arterial hypertension. Eur Respir J 2015;46:152-164.

4 Kylhammar D, Kjellström B, Hjalmarsson C, Jansson K, Nisell M, Söderberg S, Wikström G, Radegran G; SveFPH and SPAHR: A comprehensive risk stratification at early followup determines prognosis in pulmonary arterial hypertension. Eur Heart J 2017, Epub ahead of print.

5 Weatherald J, Boucly A, Chemla D, Savale L, Peng M, Jevnikar M, Jais X, Taniguchi $Y$, O'Connell C, Parent F, Sattler C, Herve P, Simonneau G, Montani D, Humbert M, Adir Y, Sitbon O: The prognostic value of follow-up hemodynamic variables after initial management in pulmonary arterial hypertension. Circulation 2018;137:693-704.

6 Galie N, Jansa P, Pulido T, Channick RN, Delcroix M, Ghofrani HA, Le Brun FO, Mehta S, Perchenet L, Rubin LJ, Sastry BKS, Simonneau G, Sitbon O, Souza R, Torbicki A: SERAPHIN haemodynamic substudy: the effect of the dual endothelin receptor antagonist macitentan on haemodynamic parameters and NT-proBNP levels and their association with disease progression in patients with pulmonary arterial hypertension. Eur Heart J 2017;38:1147-1155.
7 Galie N, Grimminger F, Grunig E, Hoeper MM, Humbert M, Jing ZC, Keogh AM, Langleben D, Rubin LJ, Fritsch A, Davie N, Ghofrani HA: Comparison of hemodynamic parameters in treatment-naive and pre-treated patients with pulmonary arterial hypertension in the randomized phase III PATENT-1 study. J Heart Lung Transplant 2017;36:509-519.

8 Boucly A, Weatherald J, Savale L, Jais X, Cottin V, Prevot G, Picard F, de Groote P, Jevnikar M, Bergot E, Chaouat A, Chabanne C, Bourdin A, Parent F, Montani D, Simonneau G, Humbert M, Sitbon O: Risk assessment, prognosis and guideline implementation in pulmonary arterial hypertension. Eur Respir J 2017;50:pii1700889.

9 Grunig E, Tiede H, Enyimayew EO, Ehlken N, Seyfarth HJ, Bossone E, D'Andrea A, Naeije R, Olschewski H, Ulrich S, Nagel C, Halank $\mathrm{M}$, Fischer C: Assessment and prognostic relevance of right ventricular contractile reserve in patients with severe pulmonary hypertension. Circulation 2013;128:2005-2015.

10 Guyatt GH, Pugsley SO, Sullivan MJ, Thompson PJ, Berman L, Jones NL, Fallen EL, Taylor DW: Effect of encouragement on walking test performance. Thorax 1984;39:818-822.

11 Thenappan T, Shah SJ, Rich S, Tian L, Archer SL, Gomberg-Maitland M: Survival in pulmonary arterial hypertension: a reappraisal of the NIH risk stratification equation. Eur Respir J 2010;35:1079-1087.

12 Gareth A, Benner A: mfp: Multivariable Fractional Polynomials. R package version 1.5.2, 2015. 
13 Williams BA, Mandrekar JN, Mandrekar SJ, Cha SS, Furth AF: Finding optimal cutpoints for continuous covariates with binary and time-to-event outcomes. Technical Report Series \#79. Department of Health Sciences Research, Mayo Clinic, Rochester, MN, June 2006. https://www.mayo.edu/research/documents/biostat-79pdf/doc-10027230.

14 Harrell FE Jr: rms: Regression Modeling Strategies. R package version 5.1-1, 2017.
15 Nickel N, Golpon H, Greer M, Knudsen L, Olsson K, Westerkamp V, Welte T, Hoeper MM: The prognostic impact of follow-up assessments in patients with idiopathic pulmonary arterial hypertension. Eur Respir J 2012; 39:589-596.

16 Benza RL, Miller DP, Gomberg-Maitland M, Frantz RP, Foreman AJ, Coffey CS, Frost A, Barst RJ, Badesch DB, Elliott CG, Liou TG, McGoon MD: Predicting survival in pulmonary arterial hypertension: insights from the Registry to Evaluate Early and Long-Term Pulmonary Arterial Hypertension Disease Management (REVEAL). Circulation 2010; 122:164-172.
17 Cook NR: Statistical evaluation of prognostic versus diagnostic models: beyond the ROC curve. Clin Chem 2008;54:17-23.

18 Boucly A, Cottin V, Nunes H, Jais X, Tazi A, Prevot G, Reynaud-Gaubert M, Dromer C, Viacroze C, Horeau-Langlard D, Pison C, Bergot E, Traclet J, Weatherald J, Simonneau G, Valeyre D, Montani D, Humbert M, Sitbon $\mathrm{O}$, Savale L: Management and long-term outcomes of sarcoidosis-associated pulmonary hypertension.EurRespirJ2017;50:pii1700465. 